\title{
Installation of pedal-operated alcohol gel dispensers with behavioral nudges and changes in hand hygiene behaviors during the COVID-19 pandemic: A hospital-based quasi-experimental study
}

\author{
Wit Wichaidit, ${ }^{1}$ Sommanas Naknual, ${ }^{2}$ Nanta Kleangkert, ${ }^{2}$ Tippawan Liabsuetrakul ${ }^{1}$ \\ ${ }^{1}$ Epidemiology Unit, Faculty of Medicine, Prince of Songkla University, Hat Yai; ${ }^{2}$ Songklanagarind Hospital, Hat \\ Yai, Thailand
}

\begin{abstract}
We conducted a quasi-experimental study and compared hand hygiene behaviors at potential pathogen transmission events among outpatient visitors (according to structured observations a trained enumerator) before and after installation of 12 pedal-operated alcohol gel dispensers with behavioral nudges (signs attached to the dispensers) at a tertiary hospital in southern Thailand during the COVID-19 pandemic. The enumerator observed 243 events during the pre-intervention period and 223 events during the postintervention period. Prevalence of hand hygiene was significantly different between the pre-intervention and post-intervention periods ( 0 vs 24 events, or $0 \%$ vs $11 \%$, respectively; p-value $<0.001$ ). However, 21 of 24 hand hygiene events were of participants who came from outside the observation area, used the dispensers, then left. Nonetheless, the intervention might have helped to increase access to hand hygiene materials and created opportunities for hand hygiene among hospital visitors in general.
\end{abstract}

\section{Introduction}

Tertiary health facilities are visited by a large number of outpatients and visitors, many of whom may harbor respiratory diseases including coronavirus disease 2019 (COVID-19). ${ }^{1}$ COVID19 can be prevented by frequently performing hand hygiene in addition to physical distancing and wearing masks..$^{2-4}$ However, hand hygiene is inadequately practiced among healthcare staff. ${ }^{5-7}$ Scarce data exists on hand hygiene behavior of outpatients and visitors at health facilities, but hand hygiene after respiratory fluid contact is infrequent in the general population. ${ }^{8}$ Interventions to increase hand hygiene behavior in hospital setting can contribute to COVID-19 prevention.

Behavioral nudges refer to changes in the physical environment or presence of physical cues to influence behaviors without direct communication, ${ }^{3}$ which can affect behavioral drivers and motivate behaviors. ${ }^{9}, 10$ Installation of alcohol gel dispensers that are easily noticeable can help to improve access and opportunities for hand hygiene, ${ }^{10}$ and use of nudges can help to motivate behaviors. No previous study has assessed changes in hand hygiene behavior in outpatient service areas before and after installation of alcohol gel dispensers and nudges. In this study, we compared the observed hand hygiene behaviors among outpatient visitors before and after installation of pedal-operated alcohol gel dispensers with behavioral nudges in outpatient service areas at a tertiary hospital in southern Thailand in the context of the COVID-19 pandemic.

\section{Design and Methods}

\section{Study design and intervention}

This was a hospital-based quasi-experimental (pre-intervention $v s$ post-intervention) study conducted at a tertiary hospital in southern Thailand. The study sites at the hospital included: i) the Internal Medicine Outpatient Department; ii) the Surgery Outpatient Department; and iii) the Pharmacy. All study sites were open to serve outpatients and visitors during the study period. The intervention in our study included installation of a pedal-operated alcohol gel dispensers with behavioral nudges (Figure 1). The alcohol gel bottle was not fixed to the platform. However, the platform could be adjusted to an appropriate distance to the top edge to tightly fit the alcohol gel bottle. Each time the foot pedal is pushed, the top edge would move down and press the beak of the alcohol gel bottle, dispensing approximately $1 \mathrm{ml}$ of alcohol gel. Depending on the user and the hand-rub technique, approximately 1-2 pushes are required for a user to perform hand hygiene.

The alcohol gel dispensers were ordered and donated by a senior management staff of the hospital upon being informed of the study. The investigators designed behavioral nudge prototypes

Significance for public health

This study compared hand hygiene behaviors after installation of pedal-operated alcohol gel dispensers compared to hand hygiene behaviors before the installation. Prevalence of observed hand hygiene was higher after installation compared to before installation, which suggested that the installation might have eliminated some barriers to hand hygiene. However, use of the alcohol gel might not necessarily indicate proper hand disinfection, and the prevalence of hand hygiene remained low, which highlighted the ongoing need to improve hand hygiene at potential pathogen transmission events (including transmission of COVID-19) at health facilities. Future studies should aim to understand the reasons(s) for missed hand hygiene opportunities and consider incorporating interventions that have shown effectiveness in improving hand hygiene behaviors among healthcare workers, as well as materials from the World Health Organization's Guidelines on hand hygiene in health care. 
after review of the literature ${ }^{9,10}$ and consultation with a graphic designer who works in the same organization as the study investigators, who then designed the prototype of the nudges. The investigators included the images of the prototypes in the pre-intervention interview questionnaire and asked respondents about their opinions on the design and interpretation of the nudges, as well as their beliefs pertaining to COVID-19 transmission, prevention and control based on the health belief model. ${ }^{11}$ On the last day of preintervention data collection, the investigators summarized the responses to the pre-intervention interview questionnaires, communicated the findings to the graphic designer, and discussed and finalized the nudge images (such as the identification of healthcare workers as the main actor to correct non-compliance to hand hygiene, hence use of the image of a nurse with the message to invite hand hygiene). The investigators then printed the nudge images in color on $180 \mathrm{gsm}$ paper, laminated the print-outs and attached the images to a plastic feature board and the alcohol gel dispensers for durability using double-faced adhesive tape. The investigators also printed the footprint images and attached them to the body of the gel dispensers in a similar manner. We installed 4 alcohol gel dispensers per study site, or 12 alcohol gel dispensers in total, at locations deemed to be most noticeable by patients and visitors. At Internal Medicine Outpatient Department and Surgery Outpatient Department, we installed the gel dispensers near the blood pressure measurement areas and the patients' waiting areas in consultation with the nurses in the Department. At the Pharmacy, we installed the gel dispensers near the columns between the patients' waiting area and the pharmacy windows. We checked the dispensers after the end of service (approximately 5 $\mathrm{pm}$ ) each day during the study period and replenish the alcohol gel bottles that were more than half-empty.

\section{Enumerator training and data collection}

Before we began pre-intervention data collection, we trained three freelance research assistants from outside the hospital and the university as enumerators. All enumerators were trained in questionnaire interview. One of the three enumerators was trained on how to conduct structured observation with table-top exercises and on-the-job training. On each day of data collection, each enumerator was randomly assigned to one of the three study sites. The enumerator who was tasked with structured observation was instructed to find a location where outpatients and visitors to the site could be observed, at an adequate distance and in a surreptitious manner to avoid reactivity, and record hand hygiene behaviors at all potential pathogen transmission events during the observation period using the KoboCollect application on Android phone. We instructed the enumerator to record only the behaviors of outpatients and visitors, i.e., people who were not wearing hospital staff identification badges. The enumerator would open the application on their phone and fill out an electronic questionnaire

Table 1. Characteristics of observed outpatient visitors during the pre-intervention and post-intervention periods (before and after installation of pedal-operated alcohol gel dispensers and behavioral nudges).

\begin{tabular}{|c|c|c|c|}
\hline & $\begin{array}{l}\text { Pre-intervention } \\
\text { ( } n=243 \text { events) }\end{array}$ & $\begin{array}{l}\text { Post-intervention } \\
\text { (n=223 events) }\end{array}$ & p-value* \\
\hline $\begin{array}{l}\text { Observed location } \\
\text { General Medicine Outpatient Department } \\
\text { Surgery Outpatient Department } \\
\text { Pharmacy }\end{array}$ & $\begin{array}{l}82(33.7 \%) \\
70(28.8 \%) \\
91(37.4 \%)\end{array}$ & $\begin{array}{l}70(31.4 \%) \\
77(34.5 \%) \\
76(34.1 \%)\end{array}$ & N/A \\
\hline $\begin{array}{l}\text { Age group of observed people (as estimated by the observer) } \\
\text { Preschooler (1-5 years) } \\
\text { Primary school age (6-12 years) } \\
\text { Secondary school age (13-17 years) } \\
\text { Adult (18-59 years) } \\
\text { Elderly ( } 60 \text { years and older) }\end{array}$ & $\begin{array}{c}3(1.2 \%) \\
3(1.2 \%) \\
3(1.2 \%) \\
127(52.3 \%) \\
107(44.0 \%)\end{array}$ & $\begin{array}{c}3(1.2 \%) \\
4(1.8 \%) \\
1(0.4 \%) \\
148(66.4 \%) \\
69(30.9 \%)\end{array}$ & N/A \\
\hline $\begin{array}{l}\text { Sex of observed people (as assumed by the observer) } \\
\text { Male } \\
\text { Female }\end{array}$ & $\begin{array}{c}86(35.4 \%) \\
157(64.6 \%)\end{array}$ & $\begin{array}{c}79(35.4 \%) \\
144(64.6 \%)\end{array}$ & N/A \\
\hline $\begin{array}{l}\text { Potential pathogen transmission event } \\
\text { Respiratory fluid contact (coughing, sneezing, others) } \\
\text { Touching face with bare hands (eyes, nose, mouth, other parts of the face) } \\
\text { Touching mask or face cover } \\
\text { Eating or drinking }\end{array}$ & $\begin{array}{c}9(3.7 \%) \\
77(31.7 \%) \\
116(47.7 \%) \\
41(16.9 \%)\end{array}$ & $\begin{array}{c}5(2.2 \%) \\
52(23.3 \%) \\
104(46.6 \%) \\
41(18.4 \%)\end{array}$ & $\begin{array}{l}0.515 \\
0.056 \\
0.885 \\
0.759\end{array}$ \\
\hline $\begin{array}{l}\text { Other events }{ }^{\circ} \\
\text { Hand hygiene behavior } \\
\text { Hands not washed } \\
\text { Hand(s) washed }<20 \text { seconds } \\
\text { Hand(s) washed } \geq 20 \text { seconds } \\
\text { Hand(s) washed, unsure regarding time } \\
\text { Location where hand hygiene was performed } \\
\text { Used own hand sanitizer tube } \\
\text { At the hospital-provided alcohol dispenser } \\
\text { At the installed pedal-operated alcohol dispenser } \\
\end{array}$ & $\begin{array}{c}0(0.0 \%) \\
243(100.0 \%) \\
0(0.0 \%) \\
0(0.0 \%) \\
0(0.0 \%) \\
\text { N/A } \\
\text { N/A } \\
\text { N/A } \\
\text { N/A }\end{array}$ & $\begin{array}{c}21(9.4 \%) \\
199(89.2 \%) \\
13(5.8 \%) \\
9(4.0 \%) \\
2(0.9 \%) \\
(\mathrm{n}=24 \text { events) } \\
1(4.2 \%) \\
1(4.2 \%) \\
22(91.7 \%) \\
\end{array}$ & $\begin{array}{c}<0.001 \\
<0.001 \\
<0.001 \\
0.005 \\
0.441 \\
\text { N/A }\end{array}$ \\
\hline
\end{tabular}

${ }^{*}$ As assessed by chi-square test of independence; 'including hospital visitors who approached the alcohol dispensing unit from outside the observation area, obtain hand hygiene gel to wash their hands, then left the observation area; N/A, not available. 
on the event that he or she had just observed. At the end of day each day, the data on the phone were then uploaded to a secured data hosting website, which the investigators could then download and analyze. Potential pathogen transmission events included: 1) respiratory fluid contact (coughing, sneezing, any other type of contact with respiratory fluid such as feeding one's children or wiping their nose); 2) touching face with bare hands (touching the eyes, nose, mouth, or other parts of the face); 3) touching one's mask or face cover; 4) eating or drinking. Enumerators were also asked to record all observed hand hygiene behaviors that occurred despite not being able to identify the pathogen transmission event and record the event as "5) hand hygiene at other events", including hand hygiene by visitors from outside the enumerator's field of vision. If there were more than one events happening at the same time, the enumerator was to make a mental note of the observed events and enter the details of each event separately. For each potential pathogen transmission event, the enumerator would record whether the event was accompanied by hand hygiene behavior. When hand hygiene behavior was observed, the enumerator also recorded whether hand hygiene event lasted 20 seconds or longer, and the location where hand hygiene was performed.

The enumerator conducted pre-intervention observations on 15-18 June 2020, we installed the alcohol gel dispensers and nudges on 20-21 June 2020, and the enumerator conducted postintervention observations on 22-24 June 2020. Structured observations were made generally between 10:00 am to 2:30 pm.

\section{Results}

The enumerator observed a total of 243 potential pathogen transmission events in the pre-intervention period and 223 events during the post-intervention period, most of which were act of touching one's mask or face cover (Table 1). Pre-intervention and post-intervention events were similar to one another with regard to the location where the event occurred and the sex ratio of individuals observed. However, post-intervention observations included fewer events involving elderly persons. Post-intervention observations also included fewer events involving touching face with bare hands. The enumerator did not observe any hand hygiene at potential pathogen transmission during the pre-intervention period,

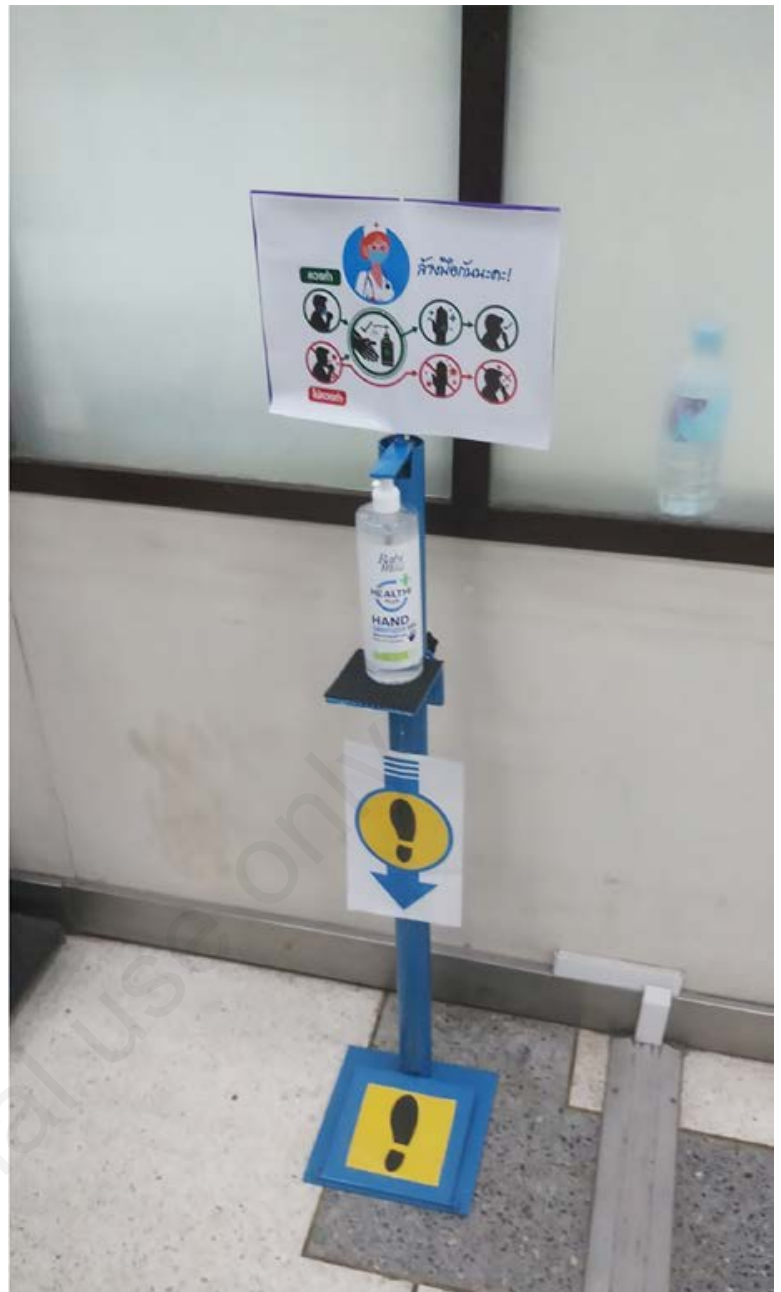

Figure 1. Example of pedal-operated alcohol gel dispensers with behavioral nudges (the sign at the top, on the stem, and at the pedal). On the sign at the top, the text in blue says "Let's wash our hands", the text in green says "Should do", and the text in red says "Should not do".

Table 2. Observed hand hygiene behaviors among outpatient visitors after installation of hand hygiene stations and behavioral nudges by type of transmission events and location.

$\begin{array}{cccc}\begin{array}{c}\text { Hands not } \\ \text { washed }\end{array} & \begin{array}{c}\text { Hand(s) } \\ \text { washed }<20 \\ \text { seconds }\end{array} & \begin{array}{c}\text { Hand(s) } \\ \text { washed } \geq 20 \\ \text { seconds }\end{array} & \begin{array}{c}\text { Hand(s) } \\ \text { washed, unsure } \\ \text { regarding time }\end{array}\end{array}$

Potential pathogen transmission event

Respiratory fluid contact (coughing, sneezing, others) ( $\mathrm{n}=17$ events)

$17(100.0 \%) \quad 0(0.0 \%) \quad 0(0.0 \%) \quad 0(0.0 \%)$

Touching face with bare hands (eyes, nose, mouth, other parts of the face) (n=52 events) $51(98.1 \%) \quad 1(1.9 \%) \quad 0(0.0 \%) \quad 0(0.0 \%)$

Touching mask ( $\mathrm{n}=104$ events)

Eating or drinking ( $\mathrm{n}=41$ events)

$103(99.0 \%) \quad 1(1.0 \%) \quad 0(0.0 \%) \quad 0(0.0 \%)$

Other events* (n=21 events)

$40(97.6 \%) \quad 1(2.4 \%) \quad 0(0.0 \%) \quad 0(0.0 \%)$

Observed location

General Medicine Outpatient Department ( $\mathrm{n}=70$ events)

$0(0.0 \%) \quad 10(47.6 \%) \quad 9(42.9 \%) \quad 2(9.5 \%)$

Surgery Outpatient Department ( $\mathrm{n}=77$ events)

\begin{tabular}{llll}
$64(91.4 \%)$ & $3(4.3 \%)$ & $2(2.9 \%)$ & $1(1.4 \%)$ \\
$73(94.8 \%)$ & $3(3.9 \%)$ & $1(1.3 \%)$ & $0(0.0 \%)$ \\
$62(81.6 \%)$ & $7(9.2 \%)$ & $6(7.9 \%)$ & $1(1.3 \%)$ \\
\hline
\end{tabular}

Pharmacy ( $\mathrm{n}=76$ events)

*Including hospital visitors who approached the alcohol dispensing unit from outside the observation area, obtain hand hygiene gel to wash their hands, then left the observation area. 
whereas there were 24 hand hygiene events during post-intervention observations, of which 22 events happened at the installed alcohol dispensers, and 9 events lasted 20 seconds or longer.

More detailed analyses showed that 21 of 24 observed hand hygiene events were of patients and visitors from outside the observation area who used the dispensers to perform hand hygiene, then left the observation area. During post-intervention observations, there was no hand hygiene after respiratory fluid contact, and prevalence of hand hygiene after touching face with bare hands, after touching mask, and before eating or drinking were all between $1 \%$ to $3 \%$. Hand hygiene was more commonly observed at the pharmacy than at the outpatient departments (Table 2).

\section{Discussion}

We investigated changes in hand hygiene behaviors according to structured observations before and after installation of alcohol gel dispensers with behavioral nudges in outpatient service areas at a tertiary hospital amidst the COVID-19 pandemic in mid-June 2020 during weekdays. Although hand hygiene behaviors significantly differed between the pre-intervention and post-intervention periods, most of the observed hand hygiene events were not of visitors within the observed areas, but other visitors who passed by the installed dispensers and used the device to perform hand hygiene. Furthermore, studies suggested that use of alcohol hand rub may not necessarily indicate proper hand disinfection, ${ }^{12,13}$ thus caveat is advised in the interpretation of the study findings. There were 2 events involving alcohol gel dispenser use where the length of hand hygiene was unknown. Future studies should consider better observation protocols to avoid such missing information. The overall prevalence of hand hygiene in this study was lower than previous report in a high-income country, ${ }^{10}$ although in our study the dispensers were placed within the waiting areas whereas in the mentioned study the dispenser was placed at the entrance to an ICU.

During both the pre-intervention and post-intervention periods, the study hospital also had alcohol hand sanitizers available for visitors to use in addition to the alcohol gel at our dispensers. These hand sanitizers were in white, non-labeled plastic bottles at the nurses or pharmacists' desks, which might be difficult for visitors to notice or visitors might have felt uncertain about whether they were allowed to use the hand sanitizers. Using the FOAM theoretical framework, ${ }^{14}$ we posit that installation of our dispensers, which were noticeable in design with a sign that invited use for hand hygiene, might have reduced these barriers and provided opportunities and materials for hand hygiene among outpatients and visitors in general. The observed difference in hand hygiene prevalence may be of public health importance, given the non-negligible risk of nosocomial infection of COVID-19 ${ }^{1}$ and the importance of hand hygiene in reduction of COVID-19 transmission. ${ }^{15}$ The observed hand hygiene behavior after installation of the dispensers and nudges also provided evidence regarding feasibility of the use of such devices in healthcare setting in middle-income countries. In that regard, we did not investigate the dispensing performance of the pedal-operated dispensers, nor did we alter the hand rub's composition or other components, which could have yielded higher dispenser usage. ${ }^{16}$ The devices used in this study might have been less efficient and more prone to spraying than other foot-operated devices currently available.

The low number of people performing hand hygiene seemed to be inconsistent with the situation of COVID-19. The lack of hand hygiene after respiratory fluid contact is concerning given the mode of transmission of coronavirus. ${ }^{17}$ More effective intervention is needed to motivate hand hygiene at potential pathogen transmission events. Social norms-based messages that emphasize possible sanctions from healthcare workers can lead to resentment, thus considerations for use of norms-based messages should be made with care. ${ }^{18}$ Future studies should aim to understand the reason(s) for missed hand hygiene opportunities (e.g., unfamiliarity with alcohol gel, uncertainty regarding the permission to use dispensers, lack of awareness of hand hygiene, social desirability to not use the dispensers) and attempt to address them in subsequent iterations. Interventions that had shown effect among hospital visitors and healthcare workers, such as installation of additional inconspicuous visual nudges, ${ }^{19}$ less detailed but more visible signs with norms-based message ${ }^{10}$ could be considered. In addition, images from the World Health Organization's Guideline on Hand Hygiene in Health Care pertaining to hand hygiene technique with alcoholbased formulation ${ }^{20}$ should also be incorporated into future interventions in order to increase effectiveness in reducing pathogen transmission.

Our study is one of the few intervention studies to assess changes in hand hygiene behaviors before and after installation of alcohol gel dispensers and nudges among outpatients and visitors using structured observations. However, a number of limitations should be taken into consideration. Firstly, it was not possible to blind the enumerator to the intervention status. If the possibility of observer bias was non-negligible, then the prevalence of hand hygiene might have been under-estimated or over-estimated at preintervention and post-intervention periods, respectively. Secondly, at Internal Medicine Outpatient Department and Surgery Outpatient Department, it was not possible to observe the entire service area from a single location, and the enumerator was allowed to move to gain the best view of the visitors. The convenience-based relocations might have biased the findings from these two locations. Thirdly, due to time and resources constraints, we collected post-intervention only on 3 consecutive days after installation of the alcohol gel dispensers and nudges. The post-intervention findings cannot be generalized beyond the time and setting of the study.

\section{Conclusion}

In conclusion, in this quasi-experimental study, we installed pedal-operated alcohol gel dispensers with nudges designed according to data and feedback provided by the potential target population. There was no observed hand hygiene in the outpatient service areas before the installation. After the installation, there was a very small increase in prevalence of hand hygiene at potential pathogen transmission events in the target population, but there was a notable increase in hand hygiene among those from outside the field of observation who used the alcohol gel dispensers and left. The findings suggested that the installation helped to increase access to hand hygiene materials and opportunities for hand hygiene, which in turn might have helped to reduce probability of infection with COVID-19 and other respiratory diseases by increasing overall hand hygiene. The findings also provided evidence for feasibility of the use of alcohol gel dispensers with nudges in hospital setting in middle-income countries. Nonetheless, more effective intervention is needed to motivate hand hygiene at potential pathogen transmission events. 
Correspondence: Wit Wichaidit, Epidemiology Unit, 6th Floor, Faculty of Medicine Administration Building, Prince of Songkla University, 15 Karnjanavanich Rd., Hat Yai District, Songkhla Province 90110, Thailand. Tel. +66.74.451165 - Fax: +66.74.429754. E-mail: wit.w@psu.ac.th

Key words: COVID-19; hand hygiene; hospital; intervention; patients.

Contributions: WW, TL, conceptualization, methodology; SN, NK, setting up study sites; WW, TL, SN, NK, training enumerators, intervention delivery; WW, data management, formal analysis, original draft preparation; WW, TL, funding acquisition; writing - original draft preparation; WW, TL, SN, NK, manuscript review and editing.

Acknowledgements: We would like to thank our enumerators and staff of the study sites for their assistance and cooperation. We also would like to thank the hospital management staff who donated the alcohol gel dispensers to the project.

Conflict of interest: The authors have no conflicts of interest associated with the material presented in this paper.

Funding: Funding has been provided by the Faculty of Medicine, Prince of Songkla University.

Ethical approval and content to participate: The study was approved by the Human Research Ethics Committee of the Faculty of Medicine, Prince of Songkla University (REC. 63-233-19-2).

Hand hygiene behaviors at the study sites were considered to be public behaviors and structured observations did not violate privacy and confidentiality, thus the investigators were allowed an exemption from obtaining informed consent.

Availability of data and materials: The data used to support the findings of this study are available from the corresponding author upon request.

Received for publication: 16 July 2020.

Accepted for publication: 28 September 2020 .

oCopyright: the Author(s), 2020

Licensee PAGEPress, Italy

Journal of Public Health Research 2020;9:1863

doi:10.4081/jphr.2020.1863

This work is licensed under a Creative Commons Attribution NonCommercial 4.0 License (CC BY-NC 4.0).

\section{References}

1. Wong SCY, Kwong RT-S, Wu TC, et al. Risk of nosocomial transmission of coronavirus disease 2019: an experience in a general ward setting in Hong Kong. J Hosp Infect 2020;105: 119-27.

2. Biswas D, Ahmed M, Roguski K, et al. Effectiveness of a behavior change intervention with hand sanitizer use and respiratory hygiene in reducing laboratory-confirmed influenza among schoolchildren in Bangladesh: A cluster randomized controlled trial. Am J Trop Med Hyg 2019;101:1446-55.

3. Global Handwashing Partnership [Internet]. Incorporating nudges into COVID-19 communication and prevention strategies. Accessed on: 19 August 2020. Available from: https://globalhandwashing.org/incorporating-nudges-into-covid-19communication-and-prevention-strategies/
4. Greenhalgh T, Schmid MB, Czypionka T, et al. Face masks for the public during the covid-19 crisis. BMJ 2020;369:m1435.

5. Shobowale EO, Adegunle B, Onyedibe K. An assessment of hand hygiene practices of healthcare workers of a semi-urban teaching hospital using the five moments of hand hygiene. Niger Med J 2016;57:150-4.

6. Chavali S, Menon V, Shukla U. Hand hygiene compliance among healthcare workers in an accredited tertiary care hospital. Indian J Crit Care Med 2014;18:689-93.

7. Musu M, Lai A, Mereu NM, et al. Assessing hand hygiene compliance among healthcare workers in six Intensive Care Units. J Prev Med Hyg 2017;58:E231-7.

8. Wichaidit W, Biswas S, Begum F, et al. Effectiveness of a large-scale handwashing promotion intervention on handwashing behaviour in Dhaka, Bangladesh. Trop Med Int Health 2019;24:972-86.

9. Dreibelbis R, Kroeger A, Hossain K, et al. Behavior change without behavior change communication: Nudging handwashing among primary school students in Bangladesh. Int J Environ Res Public Health 2016;13:1-7.

10. iNudgeyou The Applied Behavioral Science Group [Internet]. Nudging hospital visitors' hand hygiene compliance. 2016. Accessed on: 19 August 2020. Available from: https://inudgeyou.com/en/nudging-hospital-visitors-hand-hygiene-compliance/

11. Conner M. Health behaviors. In: Smelser NJ, Baltes PB, editors. International encyclopedia of the social \& behavioral sciences. Oxford: Pergamon; 2001. p. 6506-12.

12. Lehotsky Á, Szilágyi L, Demeter-Iclănzan A, et al. Education of hand rubbing technique to prospective medical staff, employing UV-based digital imaging technology. Acta Microbiol et Immunol Hung 2016;63:217-28.

13. Szilagyi L, Haidegger T, Lehotsky A, et al. A large-scale assessment of hand hygiene quality and the effectiveness of the "WHO 6-steps." BMC Infecti Dis 2013;13:249.

14. Coombes Y, Devine J. Introducing FOAM: A framework to analyze handwashing behaviors to design effective handwashing programs. Water and Sanitation Program; 2010. Available from: https:/www.wsp.org/sites/wsp.org/ files/publications/ WSP_IntroducingFOAM_HWWS.pdf

15. Yang C. Does hand hygiene reduce SARS-CoV-2 transmission? Graefes Arch Clin Exp Ophthalmol 2020;258:1133-4.

16. Bánsághi S, Soule H, Guitart C, et al. Critical reliability issues of common type alcohol-based handrub dispensers. Antimicrob Resist Infect Control 2020;9:90.

17. Jayaweera M, Perera H, Gunawardana B, Manatunge J. Transmission of COVID-19 virus by droplets and aerosols: A critical review on the unresolved dichotomy. Environ Res 2020;188:109819.

18. Bicchieri C. Norms in the wild: how to diagnose, measure and change social norms. New York: Oxford University Press; 2017. 239 p.

19. King D, Vlaev I, Everett-Thomas R, et al. "Priming" hand hygiene compliance in clinical environments. Health Psychol 2016;35:96-101.

20. Pittet D, Allegranzi B, Boyce J, et al. The World Health Organization Guidelines on Hand Hygiene in Health Care and Their Consensus Recommendations. Infect Control Hosp Epidemiol 2009;30:611-22. 